\title{
Acceso universal al Programa de VIH/SIDA de la Ciudad de México: resultados a seis años
}

\author{
Carmen Soler Claudín, M en C. ${ }^{(I)}$
}

\begin{abstract}
Soler-Claudín C. Acceso universal al Programa de VIH/SIDA de la Ciudad de México: resultados a seis años. Salud Publica Mex 2009;5 I:26-33.
\end{abstract}

\begin{abstract}
Resumen
Objetivo. Analizar los resultados del Programa de Medicamentos Antirretrovirales Gratuitos del Programa de VIH/SIDA de la Ciudad de México (PVSCM) en la población afectada del Distrito Federal. Material y métodos. Se sistematizaron y analizaron datos de la Coordinación del PVSCM sobre atención médica, tratamiento antirretroviral (ARV) y resultados de laboratorio especializado en un análisis retrospectivo del periodo 200 I-2006, para evaluar su efecto en los pacientes atendidos. Resultados. Se incluyen datos de 5 I 46 pacientes que recibieron tratamiento ARV.Al final del periodo, $74 \%$ de ellos permanecía vigente, $12.1 \%$ se perdió en el seguimiento y $13.9 \%$ había fallecido. Conclusiones. En el Distrito Federal, durante el periodo evaluado se logró la ampliación de cobertura, eficacia en el tratamiento ARV e incremento de la sobrevida de los pacientes.
\end{abstract}

Palabras clave: acceso; antirretrovirales; atención; México

\author{
Soler-Claudín C. \\ Universal access, six years results \\ in the Mexico City HIVIAIDS Program.
}

Salud Publica Mex 2009;5 I:26-33.

\section{Abstract}

Objective. To analyze the results of the Free Antiretroviral Medication Program of the Mexico City HIVIAIDS Program among the affected population in Mexico City. Material and Methods. A retrospective analysis was conducted of medical attention, antiretroviral treatment and specialized laboratory results data from 200 I to 2006 from patients who sought services from the Mexico City HIVIAIDS Program. Results. Data from 5 I 46 patients who were undergoing ARV are presented.At the end of the period studied, $74 \%$ were current, $12.2 \%$ were not found for follow-up and $13.9 \%$ had died.

Conclusions. During the period evaluated, wider coverage was achieved in Mexico City as well as increased efficiency in ARV treatment and increased patient survival.

Key words: access; antiretrovirals; attention, Mexico
E n el año 2000, los habitantes del DF distribuidos en 16 delegaciones políticas representaban $8.8 \%$ de la población nacional. El DF tiene el menor índice de crecimiento del país ( $0.23 \%$ en promedio) y es la entidad con mayor porcentaje de población asegurada (53.5\%). El $66.6 \%$ de su población tiene entre 15 y 64 años de edad;
99.8\% vive en comunidades con más de 2500 habitantes y sólo $1.83 \%$ habla una lengua nativa. ${ }^{1}$

En la Ciudad de México se ha concentrado la mayor proporción de la epidemia de SIDA, con 13087 casos acumulados y notificados en el Registro Nacional hasta el año 2000, para un total nacional de 46601 casos. Como

(I) Departamento de Medicina Genómica y Toxicología Ambiental, Instituto de Investigaciones Biomédicas. Universidad Nacional Autónoma de México. México

Fecha de recibido: 26 de noviembre de 2007 - Fecha de aceptado: 27 de agosto de 2008 Solicitud de sobretiros: Mtra. Carmen Soler Claudín. Instituto de Investigaciones Biomédicas. Circuito Interior, Ciudad Universitaria. 04310 Delegación Coyoacán, México DF, México. Correo electrónico: csoler@biomedicas.unam.mx 
entidad federativa, es la más afectada por la epidemia, con una tasa de incidencia acumulada hasta el año 2000 de 152.1 por cada 100000 habitantes, contra 37.7 en los otros 31 estados del país (47.8, nacional). ${ }^{2}$

El sistema de salud del Gobierno del Distrito Federal (GDF) está constituido por dos instancias: la Secretaría de Salud del Distrito Federal (SSDF) y los Servicios de Salud Pública del Distrito Federal (SSPDF), un organismo público descentralizado. La SSDF no tiene competencia sobre los servicios de salud de la seguridad social ni sobre los hospitales e institutos de salud federales, incluso si éstos se encuentran en la ciudad.

La política de salud del GDF a partir del año 2001 se sustentó en el reconocimiento del valor intrínseco igual de todos los hombres y mujeres y se comprometió también a respetar y proteger la vida de cada ser humano. Este valor se concretó en el derecho ciudadano a la salud y por tanto concebido como responsabilidad gubernamental. ${ }^{3}$

En términos históricos, la atención a personas con $\mathrm{VIH} / \mathrm{SIDA}$ en México se concentró en hospitales de tercer nivel de las instituciones del sector salud nacional. De manera inicial, el tratamiento se limitaba al manejo de infecciones oportunistas y neoplasias. Fue hasta 1992 que las instituciones de seguridad social empezaron la lenta introducción de medicamentos ARV, si bien la población no asegurada (cerca de 50\%) no tuvo acceso al tratamiento hasta que en diciembre de 1997 se creó un fideicomiso (FONSIDA) que ofreció de manera primaria tratamiento sólo a menores de 18 años y mujeres embarazadas; en 1999 se amplió con mil tratamientos para hombres y mujeres adultos distribuidos en las 32 entidades federativas del país. ${ }^{4,5}$

El GDF instituyó en 2001 el PVSCM que incluía, entre otras acciones, prevención, atención médica, vigilancia epidemiológica y fortalecimiento institucional. Entre sus objetivos se encontraba "asegurar el acceso a la atención médica integral y universal al tratamiento antirretroviral en la población a su cargo".

En esta publicación se describe la integración del Programa de Medicamentos Antirretrovirales Gratuitos de la Ciudad de México (PMAGCM) en atención de primer nivel y el impacto que dicho programa ha tenido en la evolución de las personas no aseguradas afectadas del Distrito Federal, en el periodo comprendido entre 2001 y 2006.

\section{Material y métodos}

El Comité de Bioética del Programa de VIH/SIDA de la Ciudad de México avaló y supervisó todas las actividades realizadas por el PVSCM.

\section{Ingreso de pacientes a atención médica y tratamiento antirretroviral}

Los requisitos para recibir atención en la Clínica Especializada Condesa (CEC), dependiente del PVSCM y perteneciente a los SSPDF de la SSDF, eran ser residente del Distrito Federal, no disponer de seguridad social y estar infectado por VIH. Todos los pacientes ingresados a la clínica tenían diagnóstico de VIH confirmado por la prueba Western blot. Después de la apertura del expediente clínico, ${ }^{6}$ un médico especializado en VIH/SIDA (infectólogo o internista) valoraba a los pacientes y a continuación prescribía el tratamiento antirretroviral si el paciente tenía signos de diagnóstico clínico de SIDA, tratamiento ARV previo o $\mathrm{CD} 4$ menores de $350 \mathrm{cel} / \mu \mathrm{L}^{7-9}$ y controlaba el cuadro; asimismo, dicho especialista se encargaba de ajustar o cambiar los esquemas de tratamiento ARV.

Los pacientes integrados al tratamiento ARV recibían una sesión de asesoría impartida por personal de salud capacitado, en la cual se discutían la integración de los ARV en la vida regular del paciente, los posibles problemas, el suministro de información sobre probables efectos adversos y la importancia de seguir las instrucciones médicas. Se establecía un periodo determinado de una semana del mes para que el paciente acudiera a la consulta médica y recogiera sus medicamentos, previa firma de una carta de consentimiento. Los cambios de esquema los determinaba el clínico especialista responsable de cada paciente mediante una justificación por toxicidad, falla clínica, inmunológica o virológica, y los autorizaba el área de la Coordinación del Programa.

\section{Instituciones participantes}

Se consideró importante establecer procedimientos de colaboración con las instituciones federales (IFED) que proporcionaban atención a pacientes del programa FONSIDA: Hospital General de México (HGM), Hospital General Dr. Gea González (HGG), Instituto Nacional de Enfermedades Respiratorias (INER), Instituto Nacional de Ciencias Médicas y Nutrición (INCMN), Instituto Nacional de Cancerología (INCAN) e Instituto Nacional de Neurología y Neurocirugía (INNN). Todos los pacientes integrados por las IFED abrieron expediente clínico en la CEC. Los pacientes podían solicitar por escrito el cambio de institución de atención médica.

\section{Financiamiento de medicamentos}

A partir del año 2003, el financiamiento se distribuyó entre los dos niveles de gobierno, local y federal, 
mediante la firma anual de convenios o acuerdos de coordinación entre la SSA y la SSDF. Durante los años 2001 y 2002 los pacientes integrados al PMAGCM recibieron financiamiento del GDF, mientras que los enfermos que provenían de FONSIDA conservaron el financiamiento de la SSA. Durante 2003, el Gobierno Federal se comprometió a financiar a dos de cada tres pacientes nuevos ingresados; en 2004 y 2005 se continuó con dicho esquema de financiamiento y a partir del año 2006, ante la integración del VIH/SIDA al Fondo de Gastos Catastróficos del Sistema de Protección Social en Salud Federal, ${ }^{10-11}$ ha sido dicho fondo el proveedor del financiamiento requerido. El Comité para la Acreditación de Unidades Incorporadas al Fondo de Gastos Catastróficos del Sistema Nacional de Protección Social en Salud, en su sesión ordinaria del mes de marzo de 2006, dictaminó la acreditación de la unidad "Clínica Especializada Condesa" como Servicio de Alta Especialidad evaluado en el componente VIH/SIDA y le asignó una calificación de $100 \%$.

\section{Seguimiento de laboratorio}

A través de un convenio de colaboración entre el Instituto de Investigaciones Biomédicas de la Universidad Nacional Autónoma de México y la SSDF se conformó un laboratorio especializado en VIH/SIDA encargado de realizar los exámenes de laboratorio (Western blot, determinaciones de CD4 y cargas virales). Los análisis clínicos regulares se realizaron en el laboratorio clínico de la CEC.

\section{Resultados}

Los pacientes recibieron atención médica bajo dos tipos de sistemas: el proporcionado en la Clínica Especializada Condesa (CEC), unidad de primer nivel de atención ambulatoria, dependiente del PVSCM de la SSDF, cuyos servicios han sido totalmente gratuitos desde enero de 2001, o los servicios específicos para VIH estructurados en seis IFED de tercer nivel, cuyos servicios médicos se controlan bajo un sistema de cuotas de recuperación. En los seis años notificados se recibieron 172 solicitudes de cambio de institución de atención médica.

\section{Pacientes atendidos}

En enero del año 2001, la CEC contaba en su archivo clínico con 450 expedientes; durante el periodo del estudio (2001-2006) se integraron a la atención médica 6500 personas. Entre el 1 de junio de 2001 y el 31 de diciembre de 2006 se recibieron 4976 solicitudes médicas para integración a ARV y además se continuó la atención de 170 pacientes vigentes integrados bajo el esquema de FONSIDA en el Distrito Federal (figura 1).

Del total de pacientes, $76.9 \%$ se atendió en la CEC, $8.8 \%$ en los dos hospitales generales y $14.3 \%$ en los cuatro institutos nacionales. En términos globales, $85.8 \%$ correspondió a hombres y $14.2 \%$ a mujeres. La media de edad en hombres fue de 34.9 y en mujeres de 33.8. La vía de transmisión fue sexual en $99 \%$ de los casos, sobre todo en hombres que tienen sexo con hombres $(68 \%)$. De los pacientes atendidos, menos de $1 \%$ tuvo como riesgo el uso de drogas intravenosas.

La clasificación clínica indica que $52 \%$ de los pacientes hombres se encontraba en estadificación $C$ al momento de la solicitud de ARV, $33 \%$ en estadio B y $15 \%$ era asintomático; en cambio, para las mujeres, $36 \%$ se hallaba en C, $21 \%$ en B y $43 \%$ era asintomático.

La información recabada indica que $71 \%$ de los pacientes hombres integrados no había recibido tratamiento previo, mientras que en el caso de las mujeres esa proporción era de $77 \%$.

El valor informado por los médicos en la solicitud de ingreso para CD4 indica que $64 \%$ de los pacientes hombres y $62 \%$ de las mujeres tenían menos de 200 $\mathrm{CD} 4 / \mu \mathrm{L}$. En el caso de las determinaciones de carga viral disponibles anteriores al tratamiento, alrededor de $45 \%$ de los pacientes, se observa que dos terceras partes de los pacientes presentaban cargas mayores de $4.6 \mathrm{Log}$ (40 000).

Ciento ochenta personas (3.5\%) no completaron el trámite de ingreso, por lo cual se dispone de información de seguimiento de 4966 pacientes, que en diciembre de 2006 se encontraban en la situación detallada en el cuadro I.

En diciembre de 2006, 67.7\% de los pacientes se encontraba vigente, $6.3 \%$ se hallaba bajo atención después de su reingreso, es decir, que $74 \%$ de los sujetos recibía aún atención, $13.9 \%$ había fallecido y de $12.1 \%$ se perdió el seguimiento; no se consideran pacientes atendidos en el INCMN los que dejaron el programa a partir de diciembre de 2004.

\section{Esquemas y cambios de tratamiento}

El PMAGCM estableció el apego a la guía de tratamiento oficial vigente respecto de los esquemas de tratamiento utilizados: se reconoció una observancia mayor de $80 \%$. 7,12

Los tratamientos de primera línea recomendados por la Organización Mundial de la Salud $(\mathrm{OMS}),{ }_{,}^{8}(\mathrm{ZDV}$ $(\mathrm{d} 4 \mathrm{~T})+3 \mathrm{TC}+\mathrm{EFV}(\mathrm{NVP})$, fueron los más empleados en el programa; $57.3 \%$ de los pacientes hombres y $50.1 \%$ de las pacientes mujeres se integraron con estos esquemas. Se advierte una diferencia significativa en el uso de 

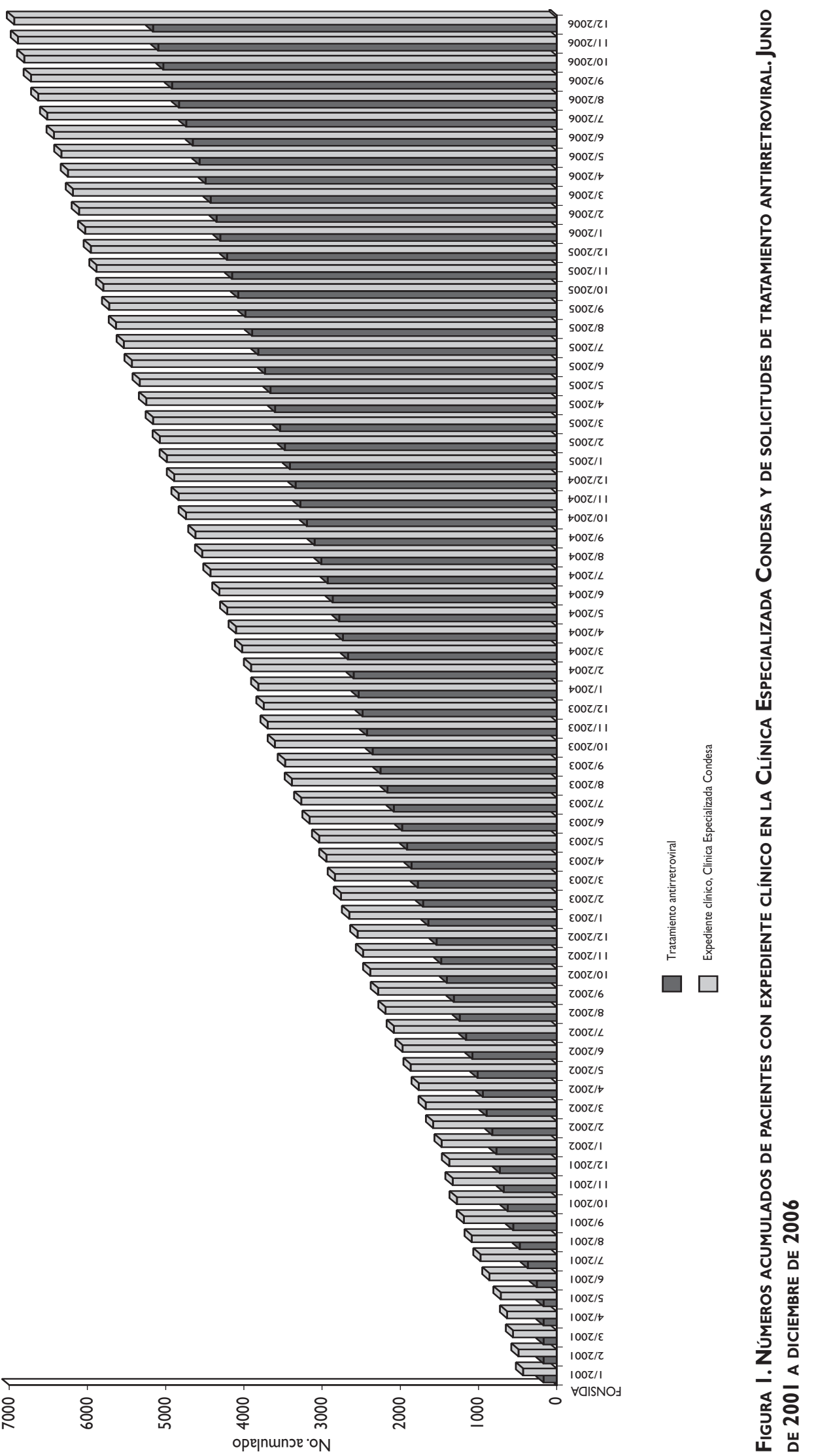


\section{Cuadro I}

Situación en diciembre de 2006 de los pacientes integrados al Programa de Medicamentos Antirretrovirales Gratuitos de la Ciudad de México (febrero de 2000 a diciembre de 2006) DE ACUERDO CON LA INSTITUCIÓN RESPONSABLE DE SU ATENCIÓN

\begin{tabular}{|c|c|c|c|c|c|c|c|c|c|c|c|c|c|c|}
\hline \multirow{3}{*}{ Vigentes } & \multicolumn{2}{|c|}{ CEC } & \multicolumn{2}{|c|}{ HGG } & \multicolumn{2}{|c|}{ HGM } & \multicolumn{2}{|c|}{ INCAN } & \multicolumn{2}{|c|}{ INCMN* } & \multicolumn{2}{|c|}{ INER } & \multicolumn{2}{|c|}{ INNN } \\
\hline & No & $\%$ & No & $\%$ & No & $\%$ & No & $\%$ & No & $\%$ & No & $\%$ & No & $\%$ \\
\hline & 2626 & 68.4 & 73 & 54.5 & 182 & 64.8 & 61 & 62.2 & 211 & 77.3 & 224 & 70.4 & 10 & 45.5 \\
\hline & 244 & 6.4 & 7 & 5.2 & 20 & 7.1 & 6 & 6.1 & 5 & 1.8 & 19 & 6 & 0 & 0 \\
\hline Fallecidos & 518 & 13.5 & 38 & 28.4 & 51 & 18.1 & 10 & 10.2 & 20 & 7.3 & 31 & 9.7 & 7 & 31.8 \\
\hline Bajas & 452 & 11.8 & 16 & 11.9 & 28 & 10 & 21 & 21.4 & 37 & 13.5 & 44 & 13.8 & 5 & 22.7 \\
\hline Total & 3840 & & 134 & & 281 & & 98 & & 273 & & 318 & & 22 & \\
\hline No completaron & 158 & 3.95 & 7 & 4.9 & 0 & 0 & 1 & I & 3 & I & 10 & 3 & I & 4.3 \\
\hline Solicitudes totales & 3998 & & $14 \mid$ & & 281 & & 99 & & 276 & & 328 & & 23 & \\
\hline $\begin{array}{l}\text { * La información de } \\
\text { CEC }=\text { Clínica Espec } \\
\text { HGG }=\text { Hospital Dr. } \\
\text { HGM= Hospital } \mathrm{Ge}\end{array}$ & $\begin{array}{l}\text { cluye } h \\
\text { idesa } \\
\text { lez } \\
\text { xico }\end{array}$ & ta dicien & re de & & & $\begin{array}{l}\text { In } \\
\text { In } \\
\text { In } \\
\text { In }\end{array}$ & $\begin{array}{l}A N=1 \\
M N=1 \\
=\operatorname{lns} \\
V=\ln \end{array}$ & $\begin{array}{l}\text { ituto } \\
\text { tituto } \\
\text { ito } \mathrm{NaC} \\
\text { uto } \mathrm{Na}\end{array}$ & $\begin{array}{l}\text { cional } \\
\text { cional } \\
\text { nal de } \\
\text { onal d }\end{array}$ & $\begin{array}{l}\text { Cance } \\
\text { Cienc } \\
\text { ferme } \\
\text { Jeurol }\end{array}$ & $\begin{array}{l}\text { logía } \\
\text { Médic } \\
\text { les } \mathrm{Res} \\
\text { a y } \mathrm{Ne}\end{array}$ & $\begin{array}{l}\text { y Nuth } \\
\text { ratoria } \\
\text { ocirugi }\end{array}$ & & \\
\hline
\end{tabular}

estos esquemas entre pacientes vírgenes al tratamiento y aquellos que habían recibido tratamiento previo (68 y $33.7 \%$, respectivamente); en relación con el uso de los inhibidores de proteasa en el esquema de integración, en $28 \%$ de los hombres y en $29 \%$ de las mujeres se solicitaron dichos inhibidores. En total se realizaron 2240 cambios de esquema.

\section{Evolución de los pacientes por CD4 y carga viral}

Cada seis meses se efectuaron determinaciones de seguimiento. Se dispone de 13720 determinaciones de CD4 (Becton Dickinson) y 12272 de carga viral (Amplicore, Roche).

En la figura 2 se muestra la evolución, según la cual los valores de CD4 mantienen un incremento hasta los 4.5 años de tratamiento, mientras que en cuanto a la carga viral desde la primera determinación se reconoce que $70.8 \%$ de los pacientes se encuentra bajo nivel de detección, y alcanza 75\% a los 4.5 años de seguimiento.

Respecto de las fallas virológicas determinadas por valores de carga viral en individuos que ya se encontraban bajo nivel (BN; valores menores de 400 copias de RNA/mL) se registraron 115 fallas entre los seis meses y un año (9.6\% de los pacientes $\mathrm{BN}$ a los seis meses), entre los 12 y 18 meses se detectaron 123 fallas (6.7\%), entre los 18 y los 24 meses se reconocieron 83 fallas (4.9\%); entre los 24 y los 30 meses se detectaron 50 fallas (3.9\%); entre los 30 y los 36 meses se registraron 38 fallas (4.4\%); entre los 36 y los 42 meses se detectaron 14 fallas (2.5\%); y, por último, entre los 42 y los 48 meses se observaron 9 fallas $(2.8 \%)$.

\section{Tasas poblacionales de atención}

La Ciudad de México está dividida desde el punto de vista administrativo en 16 delegaciones políticas, desde 115895 habitantes en la más pequeña hasta 1820888 en la mayor y con un porcentaje de masculinidad de 45.6 a $50.5 \% \cdot{ }^{13}$ Como se muestra en la figura $3 \mathrm{~A}$, la relación entre pacientes hombres y mujeres $(\mathrm{H} / \mathrm{M})$ en tratamiento es muy variable en cada delegación de la ciudad, con un rango de 2.5 a 14 .

El porcentaje de población no asegurada no es homogéneo en la ciudad; varía de un mínimo de 34.5 a un máximo de 66.9 , que en promedio arroja $46.5 \%$ de población no asegurada. ${ }^{13}$ Con base en esta información, la tasa acumulada en el periodo de 2001 a 2006 de pacientes en tratamiento antirretroviral por cada 100000 habitantes de población, sin seguridad social, entre 15 y 64 años, de acuerdo con el lugar de vivienda, tuvo límites de 66 a 912 (tasa de 324/100 000 en la ciudad) en hombres y 19 a 100 (tasa de 51/100 000 en la ciudad) para mujeres (figura 3B).

\section{Fallecimientos}

En la cohorte completa, $60.9 \%$ falleció en el primer semestre y $13.5 \%$ en el segundo semestre, es decir, que 


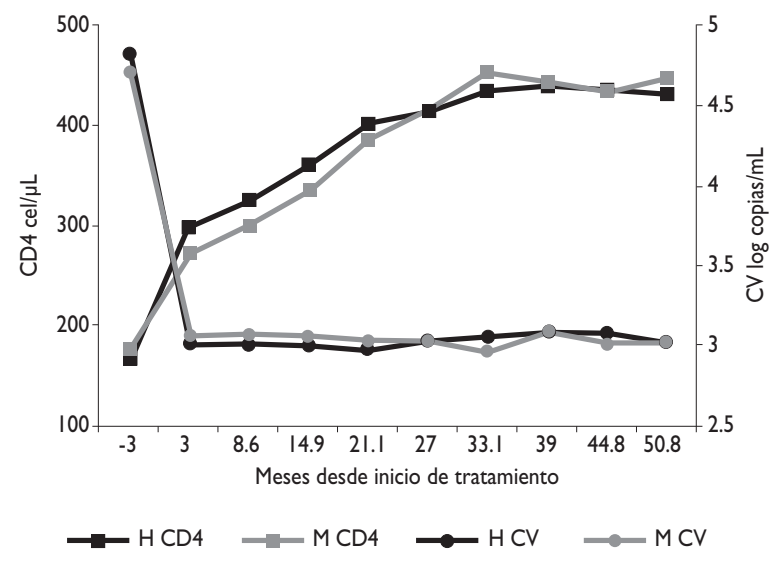

Figura 2. Media de valores de CD4/ $\mu$ L de sangre (eje IZQUIERDO) Y MEDIA DE VALORES DE LOGARITMO I 0 DE CARGA VIRAL/ML EN PLASMA (EJE DERECHO) CONTRA LA MEDIA DEL TIEMPO EN MESES DESDE EL INICIO DE TRATAMIENTO A LO LARGO DE 4.5 AÑOS DE SEGUIMIENTO EN HOMBRES Y MUJERES (JUNIO DE 200 I A DICIEMBRE DE 2006)

$74.4 \%$ pereció en el primer año de tratamiento, $13 \%$ en el segundo, $5.1 \%$ en el tercero, $3.6 \%$ en el cuarto, $2.2 \%$ en el quinto y $1.4 \%$ en el sexto.

La probabilidad de fallecer en el primer semestre después de la integración a medicamentos ARV es de $8.7 \%$ y en el segundo semestre de $2.3 \%$, lo que significa que la probabilidad de morir el primer año es de $10.9 \%$. Con posterioridad disminuye de manera considerable: el segundo año la probabilidad es de $2.4 \%$, el tercero de $1.3 \%$ y el cuarto de $1.4 \%$.

\section{Discusión}

El PMAGCM condujo a abandonar el anterior esquema de FONSIDA que, como ya se mencionó, se agotó como mecanismo posibilitador de acceso universal para población no asegurada al tratamiento en el Distrito Federal. Hasta su vigencia en el año 2001, su alcance había sido de 254 pacientes adultos del DF, de los cuales en enero de ese año se mantenían 170, cuando se hablaba ya de miles de personas afectadas.

La consolidación del servicio proporcionado en la CEC permitió incrementar la atención en el PVSCM de 40\% en 2001 a $87 \%$ en 2006 respecto de las instituciones federales que suministran atención a población abierta en el Distrito Federal. El ritmo de integración de enfermos a la atención médica y al PMAGCM se mantuvo
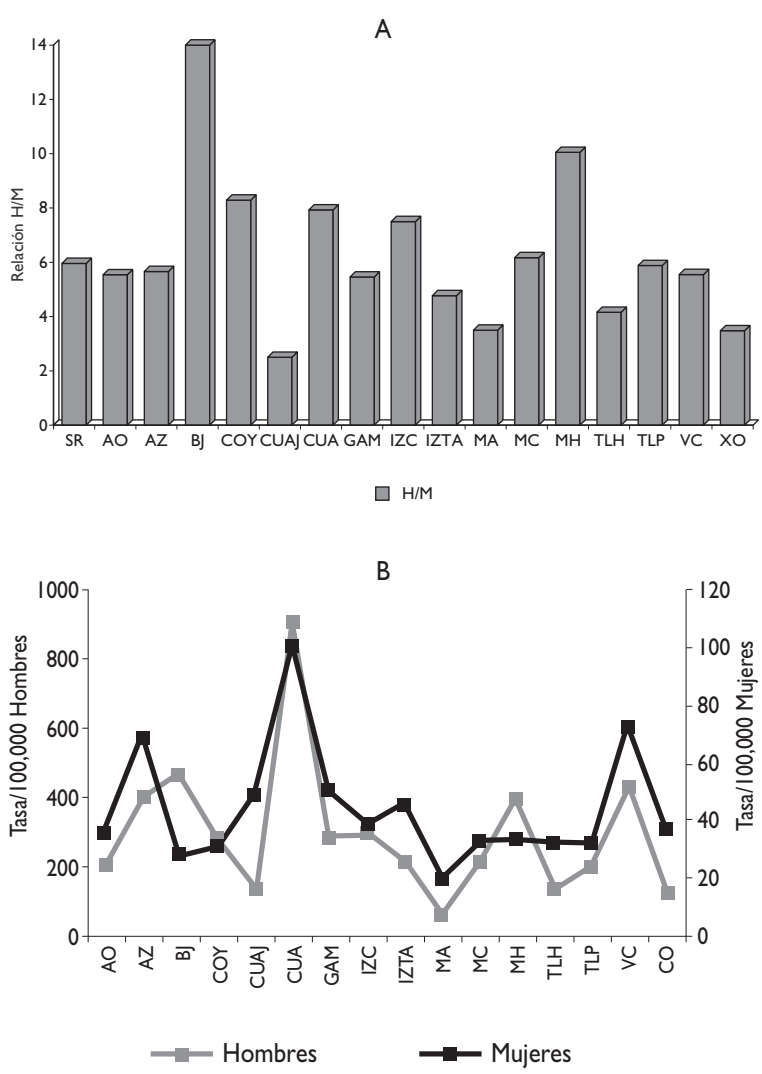

Figura 3. A relación hombre:mujer de pacientes bajo TRATAMIENTO ANTIRRETROVIRAL (200I-2006) POR DELEgación política de la Ciudad de México. B tasa de PACIENTES BAJO TRATAMIENTO POR 100000 HABITANTES NO ASEGURADOS DE I5 A 64 AÑOS POR DELEGACIÓN POLÍTICA y Sexo de la Ciudad de México. SR, sin Registro; AO, Álvaro Obregón;AZ, Azcapotzalco; BJ, Benito Juárez; COY, CoYoacán; CUAJ, CuajimalPa; CUA, Cuauhtémoc; GAM, GustaVo A. MADERO; IZC, IZTACALCO; IZTA, IZTApalapa; MA, Milpa Alta; MC, Magdalena Contreras; MH, Miguel Hidalgo;TLH,TLáhuac;TLP,Tlalpan;VC, Venustiano Carranza; $y$ XO, Xochimilco

relativamente constante a lo largo de los seis años de operación. Sin embargo, es interesante observar que a lo largo de los años se presenta una disminución ( $87.8 \%$ en 2001 a 73.5\% en 2006) de pacientes que debían integrarse al tratamiento ARV el mismo año en que abrían su expediente; en promedio, el tiempo transcurrido entre la apertura del expediente y la solicitud de ARV aumentó de modo gradual en el periodo, de 2.5 meses en 2002, a 3.2 meses en 2003, 3.4 meses en 2004, 4.8 meses en 2005 y 7.1 meses en 2006, lo que señala una mayor 
oportunidad en el diagnóstico, realizado a través de los Programas de Consejería y Diagnóstico del PVSCM, y refleja un incremento en la cobertura.

Durante el lapso de 2001 a 2002 el número de solicitudes de mujeres fue más alto de lo esperado de acuerdo con la relación de $6.03 \mathrm{H} / \mathrm{M}$ que se tiene en el global, y disminuyó la relación $\mathrm{H} / \mathrm{M}$ hasta 5.25, dato que podría interpretarse como cobertura del rezago específico de género que existía respecto del acceso al tratamiento ARV en la ciudad.

Los pacientes tenían el derecho de elegir la institución de atención y podían solicitar un cambio de ésta. $\mathrm{Al}$ analizar las instituciones de las que se solicitaron los cambios se advierte que la mayoría ocurrió hacia la CEC y el motivo expresado por los pacientes para dicho cambio fue la incapacidad de cubrir los cobros de las IFED. De no haber existido la opción de atención gratuita de CEC, un elevado número de personas hubiera abandonado su tratamiento.

Contrario a los informes de Bautista y colegas, ${ }^{14} \mathrm{el}$ apego a las guías de tratamiento vigentes en el PMAGCM es muy alto y los cambios de tratamiento son mucho menores que los señalados en ese estudio.

$\mathrm{Al}$ observar los datos oficiales de mortalidad por SIDA a lo largo de los últimos 16 años se obtiene un promedio anual de 3788 defunciones a nivel nacional, de las cuales 703 corresponden al Distrito Federal. La contribución porcentual del Distrito Federal desde 1990 hasta 2005 (últimos datos oficiales disponibles de mortalidad) varió de 33.3 a 11\%. Si se considera que la tasa de crecimiento poblacional promedio anual nacional es de $8.4 \%$ y la del Distrito Federal de $0.24 \%$, se advierte que este último ha logrado revertir la tendencia de la mortalidad hasta el punto en que se encontraba en 1990, mientras que en el país continúa en ascenso. En la figura 4 se muestran los datos de fallecimientos de nivel nacional y la tendencia en el Distrito Federal para los años que abarcan este estudio. Bautista y colaboradores ${ }^{14}$ notifican que el acceso al tratamiento en México durante 1997 a 2001 no se correlacionó con una disminución de la mortalidad, datos que se mantienen para el resto del país hasta 2005, mas no así para el Distrito Federal.

Los datos presentados indican que para ser efectivos los programas de acceso a medicamentos antirretrovirales deben considerar como parte integral el acceso de la población general a programas de detección confidencial, voluntaria y gratuita para garantizar la oportunidad de la atención médica y los tratamientos suministrados. Que las personas se integren a la atención médica antes de la necesidad de recibir medicamentos ARV representa una mayor eficacia del tratamiento ARV y un mayor incremento de la sobrevida de los pacientes.

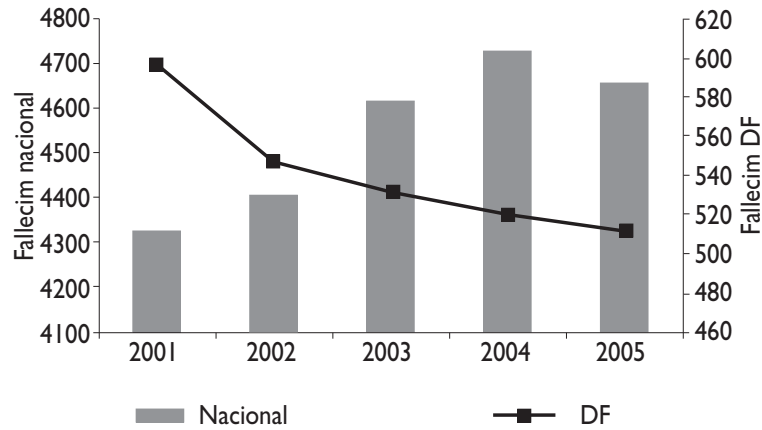

Figura 4. Fallecimientos en el Ámbito nacional de 200 I A 2005 (EJE IZQUIERDO) Y FALLECIMIENTOS EN EL Distrito Federal (eje derecho) en el mismo periodo (Fuente: INEGI/SSA/CENSIDA)

\section{Agradecimientos}

La autora expresa su agradecimiento a todos los hombres y mujeres afectados por el VIH/SIDA que recibieron atención de este equipo; a los clínicos responsables de la atención médica de la Clínica Especializada Condesa, así como de las instituciones federales; a todo el personal que laboró en el Programa de VIH/SIDA de la Ciudad de México entre 2001 y 2006; y al personal de la Unidad de Servicios en VIH/SIDA (UNAM), en especial al M. en C. Octavio Díaz Guerra por realizar los exámenes de CD4 y carga viral, a la QFB María del Carmen Basualdo por coordinar el trabajo de la Unidad de Servicios en VIH/SIDA (UNAM) y a la Dra. Yadira Palacios por sus valiosos comentarios.

\section{Referencias}

I. Instituto Nacional de Estadística, Geografía e Informática. INEGI, Censo General de Población y Vivienda 2000. Biblioteca digital. [Consultado en septiembre 2007]. Disponible en: www.inegi.gob.mx.

2. El Sida en México en el año 2000. México: CENSIDA, 2000.

3. Programa de Salud 2002-2006, Secretaría de Salud, Gobierno del Distrito Federal. Publicado en la Gaceta Oficial del Distrito Federal 2002. México: Ssa, 2002.

4. Pérez-Saleme LM, Hernández-Tepichín G. Epidemiology of HIV Infection: Twenty years of experience. Rev Inves Clin 2004;56(2): I34-I 42.

5. Río C, Sepúlveda J.AIDS in Mexico: lessons learned and implications for developing countries.AIDS 2002; 16: 1445-1457.

6. Norma Oficial Mexicana NOM-I68-SSAI-1998, del expediente clínico. 30 sep. 1998, modificada en 2003. 
7. Guía de Manejo Antirretroviral de las Personas que Viven con elVlH/ SIDA. México 2004, Secretaría de Salud. CENSIDA. 2a edición. México: Secretaría de Salud/CONASIDA, 2006.

8. World Health Organization. Scaling up antiretroviral therapy in resource limited settings. Treatment Guidelines for a Public Health Approach. Geneve:WHO, 2002

9.World Health Organization. Scaling up antiretroviral therapy in resource limited settings. Treatment Guidelines for a Public Health Approach. Revisión. Geneve:WHO, 2003.

10. Reglamento de la Ley General de Salud en Materia de Protección Social en Salud. Secretaría de Salud. Diario Oficial del 5 de abril de 2004, México. II.Acuerdo por el que se establece la Comisión para Definir

Tratamientos y Medicamentos Asociados a Enfermedades que ocasionan
Gastos Catastróficos. Consejo de Salubridad General. Diario Oficial del 28 de abril de 2004, México.

12. Guía para la Atención Médica de Pacientes con Infección porVIH/SIDA en Consulta Externa y Hospitales. 4ta ed. México: CONASIDA, 2000. 13. Instituto Nacional de Estadística, Geografía e Informática. INEGI, Censo General de Población y vivienda 2005. Biblioteca digital. [Consultado en septiembre 2007]. Disponible en: www.inegi.gob.mx. I4. Bautista-Arredondo S, Mane A, Bertozzi S. Economic impact of antiretroviral therapy prescription decisions in the context of rapid scaling-up of access to treatment: lessons from Mexico.AIDS 2006;20:101-109. 